\title{
Solutions to reduce dust emissions mass into urban air in aerated concrete production
}

\author{
Tatiana Kondratenko* \\ Don State Technical University, 344000, Rostov-on-Don, Russia
}

\begin{abstract}
Low fractional efficiency of dust separation equipment used in dust cleaning systems in the production of building materials remains one of the main causes of dust emissions with a high content of $\mathrm{PM}_{10}$ and $\mathrm{PM}_{2,5}$ fine particles in the atmospheric air, both on the industrial sites territory, and on the borders of sanitary protection zones. The wide range of building materials produced by the construction industry with different raw materials, requires a tailor-made approach to develop the solutions to reduce dust emissions. This approach is primarily based on the properties of dust emitted during the production process.
\end{abstract}

\section{Introduction}

Air quality is a challenge for one of the 17 Sustainable Development Goals (IMC), formed for international cooperation at the meeting of the UN General Assembly in 2015, and specified in the final document "Transforming our world: the 2030 Agenda for Sustainable Development".

In the frameworks of achieving IMC, the strategies are developed, plans are formed, programs are drawn up. Improving the environmental safety of construction production remains an urgent problem and relates to these strategies' implementation [1-8].

Many authors are looking for the solutions to ensure environmental safety in the production of building materials. The increased attention is dictated, first of all, by the growth of production volumes, the location of production enterprises in the immediate vicinity of the residential area.

\section{Main part}

A number of technological operations of most of the construction industry enterprises are associated with the use of raw materials in a free-flowing state, crushing, sawing, etc. That is, the process of manufacturing building materials, products and structures is accompanied by the release of dust, its entry, both into the air of working areas and into the atmospheric air. Particular attention is paid to the resulting dust-air mixture with PM10 and PM2,5 fine particles, the content of which in most cases exceeds MAD [3, 5, 8-12].

\footnotetext{
* Corresponding author: tatkondr@,rambler.ru
} 
Disperse composition and properties of dust are different depending on the production, from the raw materials used as raw materials, additives, etc.

Despite the wide range of the existing technological lines, equipment included in their composition, raw materials base, technological operations included in the production cycle for the manufacture of one or another type of building materials, dust cleaning systems, the equipment in their composition remains identical.

The results of dust content measurements in the atmospheric air at the production area boundaries as well as in the working area show the low efficiency of the existing dust collection systems and, as a consequence, significant dust emissions into the environment.

As noted earlier $[9,10,12,13]$, in order to improve the emission containment and treatment system efficiency, it is necessary to study and take into account the main properties of the generated dust (their dusting capacity, flowability, dispersed composition of dust, etc.) when developing technical solutions to ensure the fractional efficiency of dust separation equipment used in dust cleaning systems.

According to the experimental studies' results, it was found that the dust generated during the aerated concrete structures production, in terms of its dusting ability, belongs to the dusting group (50-70\%), that is, dust is characterized by the ability to pass into a suspended state, as well as a tendency to aerate, has a high dispersibility - from 45 to $88 \%$.

Rheological properties of the studied dust: good flowability - the ability to form a discrete-continuous steady flow; great aeration; weak arc formation - contributing to the hovering of dust particles over the outlet [9, 10, 12, 13]. The dust generated in the considered production is polydisperse. Also, according to the results of experimental studies, dust with a particle size of up to 20 microns enters the aspiration system. The mass fraction of the content ranges from 25 to $100 \%$ of the mass. Fraction of particles with sizes less than 10 microns (PM10) ranges from 6\% to 65\%. The above-mentioned study results of the dust dispersed composition, as well as the content of particles PM10 in the emissions and in the air, indicate a low fractional efficiency of dust collecting equipment used in dust cleaning systems (cyclones). The use of cyclones for cleaning emissions in the production of aerated concrete and aerated concrete structures gives a possibility for an excess of PM10 particles in the atmospheric air.

Experimentally obtained data on the dust content in the atmospheric air at the border of the industrial territory showed the need to search for solutions to improve the dust cleaning process efficiency, reduce dust emissions into the atmospheric air.

To solve the issue of ensuring the environmental safety of the production process for the aerated concrete production, that is, first of all, the issue of reducing dust emissions into the atmospheric air, various layouts of the dedusting unit were proposed (Figure 1, 2). The development of the schemes was carried out taking into account the study results of the operating modes of the CSF devices, previously received by the authors $[4,8,14,15]$.

In the listed works, it was found that when organizing the suction from the bunker zone of the inertial apparatus, the efficiency of the latter increases and the aerodynamic resistance decreases. It was also revealed that the CSF apparatus effectiveness increases when a dust-air flow with a lower concentration is fed to the lower inlet of the apparatus than to the upper one.

In view of the above-said, the proposed systems include two sequentially installed CSF apparatus. From the bunker zone of the second stage dust collector and the third (additional) CSF apparatus suction is organized with a smaller body diameter. To clean the dust-air mixture sucked from the first and third apparatuses, it is provided to install one more (fourth) additional dust collector with a smaller body diameter, from which, after cleaning, air is supplied to the lower inlet of the first stage dust collector. The captured dust is unloaded from the bunkers of the first and fourth apparatus and returned to the technological process [4, 13, 14, 15]. 


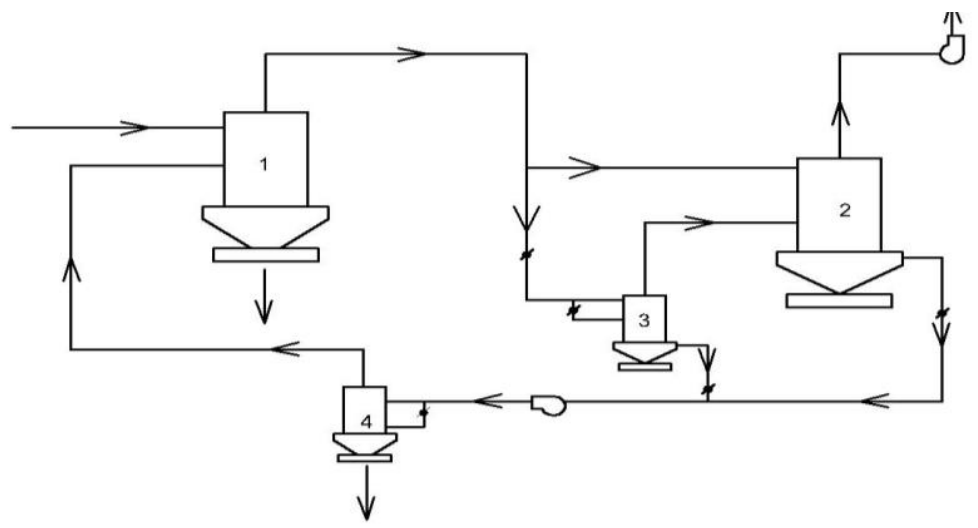

Fig. 1. The first variant of the proposed system layout for reducing dust emissions into the atmospheric air with vortex inertial apparatuses on counter swirling flows (CSF)

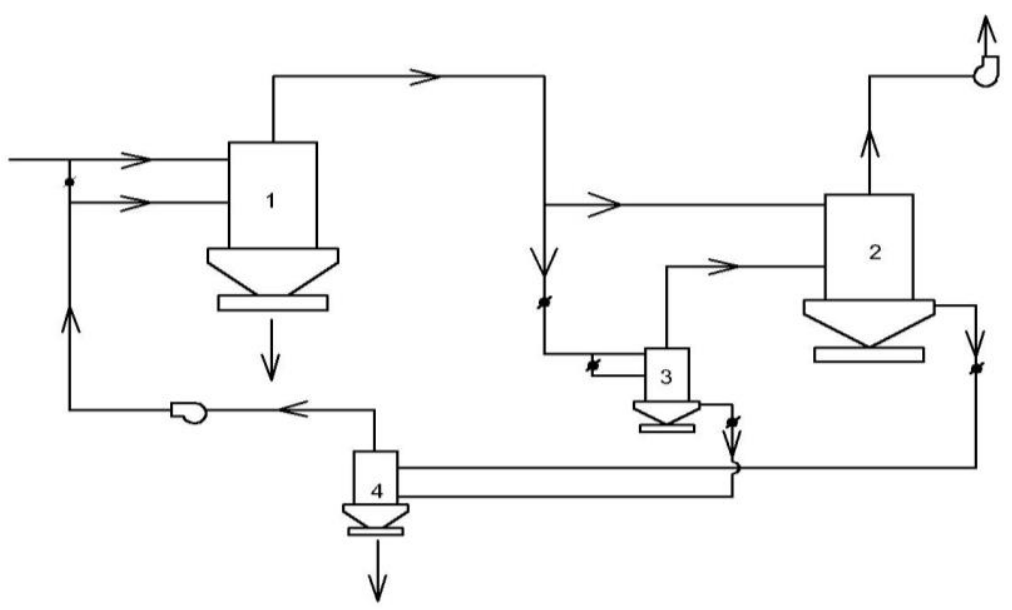

Fig.2. The second variant of the layout of the proposed system for reducing dust emissions into the air with vortex inertial apparatuses on counter swirling flows CSF

The dust-air mixture flow comes from the emission localization system to the upper inlet of the first stage vortex dust collector 1 . The flow leaving it after cleaning is divided and fed to the upper inlet of the second stage dust collector 2 and to the inlet of the additional CSF apparatus 3. Adjustment of the dust-air mixture flow rate is carried out by means of dampers. After cleaning in the apparatus 3, the flow is fed to the lower inlet of the dust collector of the second stage 2. Thus, the flows with different dust concentrations are supplied to the upper and lower inlets of the dust collector 2. After cleaning in the apparatus of the second stage 2, the air is discharged into the atmosphere. The dust-air mixture sucked from the hopper of the dust collector 2 is supplied for cleaning to the fourth additional dust collector 4, from which, after cleaning, the air is supplied to the lower inlet of the dust collector of the first stage 1 . Thus, the supply of flows with different dust contents to the upper and lower inlets of the dust collector 1 is ensured [4, 13-15].

All branch pipes are provided with the installation of dampers to regulate dust-air flows in order to achieve efficient system operation.

As shown in Figures 1 and 2, in the layout of the emission dedusting system according to the first option, it is assumed that the combined supply of 4 flows of the dust-air mixture sucked from the dust collector hoppers of the second stage 2 and the additional CSF 
apparatus 3 is assumed. When the dust cleaning unit is configured according to the second option, the recirculation flow from the apparatus 2 hopper is fed to the upper inlet of apparatus 4 , the recirculation flow from the hopper of the apparatus is fed to the lower inlet of the fourth dust collector.

\section{Conclusion}

Improving the environmental safety of a particular enterprise for the production of building materials is a complex task, the solution of which includes an analysis of the existing dust cleaning system effectiveness, the air environment state at the border of the sanitary protection zone, and the study of the emitted dust properties. The search for solutions should be based on an individual approach depending on the type of products being manufactured, the specifics of manufacturing operations, the equipment used, the characteristics of the feedstock, etc.

The proposed solutions for the system layout scheme to reduce dust emissions into the atmospheric air (in the production of aerated concrete structures) with vortex inertial apparatuses on counter swirling flows (CSF) allow:

- to increase the efficiency of the second stage apparatus, which will lead to an increase in the degree of cleaning of the entire system as a whole due to the organization of suction from the bunker zone;

- to reduce the aerodynamic resistance of the second dust collector, which will prevent a significant increase in pressure losses in the entire system due to the installation of additional equipment due to the organization of suction from the bunker zone;

- to ensure an increase in its efficiency, which, in turn, will lead to an increase in the efficiency of the system as a whole and, accordingly, to a greater decrease in dust penetration into the atmosphere due to the supply of dust-air streams with different concentrations to the upper and lower inlets of the dust collectors of the first and second stage.

\section{References}

1. V.N. Azarov, N.M. Sergina, T.O. Kondratenko, Problems of protecting urban air from pollution by industrial dust emissions, MATEC Web of Conferences.-2017 (International Science Conference on SMART City, SPbWOSCE 2016) 15-17 November 2016, Saint-Petersburg, Russia, https://www.matecconferences.org/articles/matecconf/abs/2017/20/matecconf_spbw2017_07017/matecco nf_spbw2017_07017.html

2. Problems of industrial and environmental protection: a collection of materials and scientific works of environmental engineers 6 (2014)

3. M.S. Nazarova, Problems of industrial and environmental protection: a collection of materials and scientific works of environmental engineers 6 (2014)

4. M.A. Nikolenko, Priority Research Areas: From Theory to Practice, 106-111 (2014)

5. T.O. Kondratenko, Engineering Journal of Don 4 (2), (2012) ivdon.ru/magazine/archive/n4p2y2012.

6. T.O. Kondratenko, A.V. Saybel, Engineering Journal of Don 4 (2), (2012) ivdon.ru/magazine/archive/n4p2y2012.

7. T.O. Kondratenko, Scientific review 11 (3), 815-817 (2014)

8. N.M. Sergina, M.A. Nikolenko, Engineering Journal of Don 1, (2015) 
http://www.ivdon.ru/ru/magazine/archive/n1y2015/2751

9. T.O. Kondratenko, G.M. Danilova-Volkovskaya, V.A. Fursov, T.L. Kobalia, The basic properties study of the dust particles entering the localization system and emissions cleaning in the aerated concrete production and building gas concrete blocks, IOP Publishing 698, 022075 (2019) (CATPID-2019 IOP Conf. Series: Materials Science and Engineering) 1-5 October 2019,. Kislovodsk; Russia, doi:10.1088/1757899X/698/2/022075 // https://iopscience.iop.org/article/10.1088/1757$899 \mathrm{X} / 698 / 2 / 022075$

10. T.O. Kondratenko, Experimental studies to develop the measures for reducing dust emissions mass into the atmosphere from the sources of the aerated concrete structures shop, IOP Publishing 913, 052047 (2020) (CATPID-2020 IOP Conf. Series: Materials Science and Engineering) 26-30 September 2020, Nalchik, Russia, doi: 10.1088/1757899X/913/5/052047 // https://www.scopus.com/record/display.uri?eid=2-s2.085092032215 \&origin=resultslist.

11. T.O. Kondratenko, A.P. Lapina, Assessment of the concentration and properties of dust in emissions into atmospheric air from the sources of the workshop for the aerated concrete structures production, IOP Publishing 1083, 0120812020 (2020) (CATPID2020 IOP Conf. Series: Materials Science and Engineering) 16-17 December 2020, Nalchik, Russia, doi: 10.1088/1757-899X/1083/1/012081 // https://iopscience.iop.org/article/10.1088/1757-899X/1083/1/012081

12. Investigation of the main properties of dust in the production of aerated concrete (III International scientific-practical conference "Problems and prospects of modern science) 67-73, 2014, Stavropol, Russia

13. N.M. Sergina, T.O. Kondratenko, M.A. Nikolenko, S.L. Pushenko, Principles of layout and assessment of the systems effectiveness to protect against dust pollution of air in the working area and atmospheric air, EMMFT (2017) (International Scientific Conference Energy Management of Municipal Transportation Facilities and Transport EMMFT) https://link.springer.com/chapter/10.1007/978-3-319-70987-1_75

14. A.N. Bogomolov, N.M. Sergina, T.O. Kondratenko, Procedia engineering 150, 20362041 (2016),

DOI:10/1016/g.proeng.2016.07.290, http://www.sciencedirect.com/science/article/pii/S1877705816316095?via\%3Dihub

15. T.O. Kondratenko, Scientific review 11 (2), 518-520 (2014) 